\title{
Association of Lymphocyte-to-Monocyte Ratio, Mean Diameter of Coronary Arteries, Uric Acid with Coronary Slow Flow in Isolated Coronary Artery Ectasia
}

\section{Zhuoxuan Yang}

Chinese Academy of Medical Sciences \& Peking Union Medical College Fuwai Hospital

Jiansong Yuan

Chinese Academy of Medical Sciences \& Peking Union Medical College Fuwai Hospital Jingang Cui

Chinese Academy of Medical Sciences \& Peking Union Medical College Fuwai Hospital Shubin Qiao ( $\nabla$ qsbfw@sina.com )

Chinese Academy of Medical Sciences and Peking Union Medical College Fuwai Hospital https://orcid.org/0000-0002-2956-7233

\section{Research article}

Keywords: Isolated coronary artery ectasia, Coronary slow flow, Uric acid, Mean diameter of arteries, Lymphocyte-to-monocyte ratio

Posted Date: December 7th, 2020

DOI: https://doi.org/10.21203/rs.3.rs-120664/v1

License: (1) (i) This work is licensed under a Creative Commons Attribution 4.0 International License. Read Full License

Version of Record: A version of this preprint was published at BMC Cardiovascular Disorders on March 30th, 2021. See the published version at https://doi.org/10.1186/s12872-021-01952-4. 


\section{Abstract}

Background The pathophysiology of isolated coronary artery ectasia (CAE) with coronary slow flow (CSF) phenomenon is still unclear. The purpose of this study was to investigate the risk factors for isolated CAE complicated with CSF.

Methods A total of 126 patients with isolated CAE were selected retrospectively. The patients were grouped into the no CSF(NCSF) group $(n=55)$ and CSF group $(n=71)$ according to the thrombolysis in myocardial infarction (TIMI) frame count (TFC). Data on demographics, laboratory measurements, left ventricular ejection fraction (LVEF), left ventricular end-diastolic diameter (LVEDd), TFC and diameters of three coronary arteries were collected.

Results The proportion of patients with male sex( $84.5 \%$ vs $61.8 \%, p=0.004)$ and a smoking history $(63.4 \%$ vs $43.6 \%, p=0.021)$ in the CSF group were greater than that in the NCSF group. The neutrophil-tolymphocyte ratio (NLR) $(2.44 \pm 1.12$ vs $1.89 \pm 0.58, p=0.001)$, mean diameter of coronary arteries (Mean D) ( $5.50 \pm 0.85$ vs $5.18 \pm 0.91, p<0.001)$, and uric acid (URIC) level(370.78 \pm 109.79 vs $329.15 \pm 79.71, p=0.019)$ were significantly higher in the CSF group, while the lymphocyte-to-monocyte ratio (LMR)(4.81 \pm 1.66 vs $5.96 \pm 1.75, p<0.001)$ and albumin (ALB) $(44.13 \pm 4.10$ vs $45.69 \pm 4.11, p=0.036)$ level were lower. Multivariable logistic analysis showed that the LMR(odds ratio: $0.614,95 \% \mathrm{Cl}: 0.464-0.814, p=0.001$ ), Mean D(odds ratio: $2.643,95 \% \mathrm{Cl}: 1.54-4.51, \mathrm{p}<0.001$ ) and URIC(odds ratio: 1.006, 95\%Cl:1.001-1.012, $p=0.018$ ) level were independent predictors of CSFP in CAE. The predictive power of this combination was superior to URIC and Mean D but not superior to the LMR.

Conclusions The LMR, URIC level and Mean D were independent predictors of CSF in isolated CAE. The predictive power of the LMR was not inferior to the combination of predictors.

\section{Background}

Isolated coronary artery ectasia (CAE) refers to the local diameter of a coronary artery being dilated more than 1.5 times the adjacent normal vessels and having no obvious obstruction ${ }^{[1-4]}$, with a morbidity of less than $0.32 \%{ }^{[5]}$. Coronary slow flow (CSF) phenomenon is coronary angiography that has no obvious stenosis but slow forward flow perfusion ${ }^{[6,7]}$. The incidence of CSFP in coronary angiography is less than $1 \%{ }^{[7]}$. However, it can cause serious cardiac events, such as angina pectoris, myocardial infarction, malignant arrhythmia and even sudden death ${ }^{[8]}$.

There is no consensus on the physiologic mechanism of the above two diseases. Although the change of vascular diameter will affect the velocity of flow, not all patients with CAE have CSF. Since the morbidity of these two diseases is low, large sample studies are lacking. Some small sample studies have suggested the lymphocyte-to-monocyte ratio (LMR), neutrophil-to-lymphocyte ratio (NLR), albumin (ALB) level, high-sensitivity C-reactive protein (hs-CRP) level and uric acid (URIC) level were risk factors for CSF 
or $\mathrm{CAE}^{[1,2,4,7,9-12]}$. Therefore, the aim of this study was to investigate the risk factors for CSF in patients with isolated CAE.

\section{Materials And Methods}

\section{Subjects}

From January 2010 to December 2019, 541 patients who underwent coronary angiography in our hospital and were diagnosed as having isolated CAE were selected. Inclusion criteria were as follows: the diameter of the local coronary artery was more than 1.5 times larger than that of the adjacent normal segment with the degree of stenosis $<20 \%$ and cardiac ultrasound showed that the heart structure and left ventricular ejection function (LVEF) were normal. Exclusion criteria were acute coronary syndrome, coronary spasm, coronary artery bypass graft, valve disease, congenital heart disease, Kawasaki disease, left ventricular or ventricular septal hypertrophy, immunological disease, malignant tumor, severe cerebrovascular disease, severe hepatic or renal insufficiency (creatinine $>132 \mu \mathrm{mol} / \mathrm{L}, \mathrm{AST}$ or ALT $>2$ times upper level of normal), hematological system disease or hemoglobin $<90 \mathrm{~g} / \mathrm{L}$, received steroid hormone treatment or acute inflammatory disease within 1 month. A total of 126 patients with isolated CAE were included and grouped into the CSF group $(n=71)$ and the no CSF(NCSF) group $(n=55)$.

\section{Angiography data and frame counting}

The coronary flow velocity was measured by using the thrombolysis in myocardial infarction (TIMI) frame count (TFC) according to Gibson ${ }^{[13]}$. The frame was collected at 30 frames/s, counting the number of frames from the start to the distal coronary artery. The first frame is the contrast agent completely filling the coronary artery, and the forward motion of the contrast agent can be observed. The final frame is the contrast agent reaching a certain landmark of the coronary artery. The sign of the left anterior descending (LAD) artery was the "whale tail" or "hay fork " at the distal bifurcation. The landmark of the left circumflex (LCX) artery was the distal bifurcation of the obtuse marginal branch, and the first branch of the posterolateral artery was used for the right coronary artery (RCA). Because the LAD artery is longer, the TFC for this artery should be divided by 1.7 to get a corrected TFC (CTFC). Any vessel with a cTFC $\geq$ 27 frames is defined as CSFP. The average number of TFC (Mean TFC) is obtained by summing the TFC of 3 arteries then dividing by 3 .

\section{Demographic and laboratory measurements collection}

Age, gender, body mass index (BMI), history of hypertension, type 2 diabetes mellitus, medication, smoking and drinking, white blood cell count, neutrophil count, lymphocyte count, monocyte count, red blood cell count, hemoglobin count, blood cell specific volume, red blood cell distribution width, mean platelet volume (MPV), mean red blood cell volume, $C$ reactive protein (CRP), high-sensitivity $C$ reactive protein (hs-CRP), albumin (ALB), uric acid (URIC), creatinine, total bilirubin (TBIL), indirect bilirubin (IBIL), triglycerides (TG), total cholesterol (TC), high-density lipoprotein cholesterol (HDL-C), low-density lipoprotein cholesterol (LDL-C), glycated hemoglobin (HbA1C), NT-proBNP, LVEF, and left ventricular end- 
diastolic diameter (LVEDd) were noted. The TFC and vascular diameter were also assessed by two interventional physicians.

Written informed consent was obtained from each patient included in the study, The study protocol conforms to the ethical guidelines of the 1975 Declaration of Helsinki and the study protocol has been priorly approved by the Institution's ethics committee on research on humans.

\section{Statistical analysis}

All analyses were performed using the Statistical Package for the Social Sciences version 22.0 (IBM Corp., Armonk, New York, USA) and MEDCALC (Software bvba 19, Ostend, Belgium) software programs. Continuous variables were presented as the means \pm standard deviations (normal distribution) and medias (interval changes) (non-normal distribution). Categorical variables were presented as percentages. Comparisons of categorical variables between the two groups were performed using the Chi-squared $(\chi 2)$ test. The Kolmogorov-Smirnov test was performed to assess if the variables were normally distributed. Student's t-test or Mann-Whitney $\mathrm{U}$ test was used to compare the continuous variables between the two groups according to whether they were normally distributed or not. Spearman's rho correlation analysis was performed to describe the degree of correlation between the parameters related with the mean TFC. To identify the independent predictors of CSF, univariate and multivariate logistic regression analyses (backward LR method) were performed. The variables with a $\mathrm{P}<0.1$ in the unit analysis were incorporated into the multivariate logistic regression analysis. Receiver operating characteristic (ROC) curve analysis was performed to determine additional assets of parameters found to be independent predictors of CSF. The optimal cutoff value was calculated from the point of maximum sensitivity and specificity (Youden's index). To compare the predictive performance of parameters found to be independent predictors for CSF development, the DeLong et al. test was also used. The results were evaluated within a 95\% confidence interval $(\mathrm{Cl})$ and at a significance level of $\mathrm{P}<0.05$.

\section{Results}

Comparison of the demographic and clinical characters is shown in Table 1. The proportion of males $(84.5 \%$ vs $61.8 \%, p=0.004)$ and smoking history $(63.4 \%$ vs $43.6 \%, p=0.021)$ in the CSF group was significantly higher than that in the NCSF group, and there was no significant difference in age, BMI, hypertension, type 2 diabetes mellitus, and medications between the two groups. 
Table 1

Demographic and clinical characteristics of the study population

\begin{tabular}{|llll|}
\hline & NCSF group $(\mathbf{n = 5 5 )}$ & CSF group $(\mathbf{n = 7 1 )}$ & $\boldsymbol{p}$ \\
\hline Age & $55.73 \pm 11.93$ & $55.85 \pm 11.06$ & 0.954 \\
\hline Male & $34(61.8 \%)$ & $60(84.5 \%)$ & 0.004 \\
BMI & $26.29 \pm 4.97$ & $27.15 \pm 3.41$ & 0.251 \\
\hline Smoking history & $24(43.6 \%)$ & $45(63.4 \%)$ & 0.021 \\
\hline Anti-platelet drugs & $30(54.5 \%)$ & $36(50.7 \%)$ & 0.402 \\
\hline ACElockers & $19(34.5 \%)$ & $23(32.4 \%)$ & 0.474 \\
\hline ARBs & $6(10.9 \%)$ & $5(7 \%)$ & 0.326 \\
\hline Statins & $8(14.5 \%)$ & $10(14.1 \%)$ & 0.569 \\
\hline Hypertension & $26(47.3 \%)$ & $31(43.7 \%)$ & 0.411 \\
\hline Diabetes & $36(65.5 \%)$ & $42(59.2 \%)$ & 0.296 \\
\hline LVEF & $13(23.6 \%)$ & $10(14.1 \%)$ & 0.276 \\
\hline LVEDd & $64.11 \pm 4.20$ & $48.68 \pm 4.16$ & 0.264 \\
\hline
\end{tabular}

Comparison of the laboratory variables between the two groups is shown in Table 2. The differences in TG, TC, HDL-C, LDL-C, HbA1C, LVEDd, LVEF, NT-proBNP and eGFR were not significant between the groups. The diameters of the $\operatorname{LAD}(6.27 \pm 1.25$ vs $5.52 \pm 1.33, p=0.002), \operatorname{LCX}(5.91 \pm 1.39$ vs $5.05 \pm 1.45, p$ $=0.001)$ and Mean coronary arteries $(5.50 \pm 0.85$ vs $5.18 \pm 0.91, p<0.001)$ were significantly larger in the CSF group compared to those in the NCSF group; however, no significant difference was found between the groups in RCA diameter $(6.37 \pm 2.02$ vs $5.91 \pm 1.55, p=0.173)$. The TFC in all coronary arteries(LAD: $28.43 \pm 8.16$ vs $19.45 \pm 4.81, p<0.001$; LCX:32.54 \pm 11.46 vs $20.24 \pm 4.41, p<0.001$; RCA:30.13 \pm 12.30 vs $18.74 \pm 5.02, p<0.001)$, Mean TFC(30.39 \pm 8.32 vs $19.41 \pm 3.77, p<0.001)$, $\mathrm{NLR}(2.44 \pm 1.12$ vs $1.89 \pm 0.58, p=0.001)$ and URIC(370.78 \pm 109.79 vs $329.15 \pm 79.71, p=0.019)$ level were higher in the CSF group than in the NCSF group, while the LMR $(4.81 \pm 1.66$ vs $5.96 \pm 1.75, p<0.001)$ and $\operatorname{ALB}(44.13 \pm 4.10$ vs $45.69 \pm 4.11, p=0.036)$ level were significantly lower in the CSF group compared to those in the NCSF group. 
Table 2

Comparison of laboratory variables in the study population

\begin{tabular}{|c|c|c|c|}
\hline & NCSF group $(n=55)$ & CSF group $(n=71)$ & $p$ \\
\hline LAD diameter (mm) & $5.52 \pm 1.33$ & $6.27 \pm 1.25$ & 0.002 \\
\hline LCX diameter (mm) & $5.05 \pm 1.45$ & $5.91 \pm 1.39$ & 0.001 \\
\hline RCA diameter (mm) & $5.91 \pm 1.55$ & $6.37 \pm 2.02$ & 0.173 \\
\hline Mean diameter of coronary arteries & $5.18 \pm 0.91$ & $5.50 \pm 0.85$ & $<0.001$ \\
\hline LAD TIMI frame count & $19.45 \pm 4.81$ & $28.43 \pm 8.16$ & $<0.001$ \\
\hline LCX TIMI frame count & $20.24 \pm 4.41$ & $32.54 \pm 11.46$ & $<0.001$ \\
\hline RCA TIMI frame count & $18.74 \pm 5.02$ & $30.13 \pm 12.30$ & $<0.001$ \\
\hline Mean TIMI frame count & $19.41 \pm 3.77$ & $30.39 \pm 8.32$ & $<0.001$ \\
\hline WBCs & $6.36 \pm 1.61$ & $6.60 \pm 1.58$ & 0.399 \\
\hline Neutrophils & $3.75 \pm 1.15$ & $4.11 \pm 1.22$ & 0.091 \\
\hline Lymphocytes & $2.08 \pm 0.60$ & $1.88 \pm 0.69$ & 0.08 \\
\hline Monocytes & $0.37 \pm 0.11$ & $0.41 \pm 0.13$ & 0.064 \\
\hline NLR & $1.89 \pm 0.58$ & $2.44 \pm 1.12$ & 0.001 \\
\hline LMR & $5.96 \pm 1.75$ & $4.81 \pm 1.66$ & $<0.001$ \\
\hline hsCRP & $1.53 \pm 1.67$ & $1.98 \pm 2.26$ & 0.218 \\
\hline CRP & $2.65 \pm 2.16$ & $3.33 \pm 3.34$ & 0.192 \\
\hline HGB & $147.82 \pm 14.46$ & $152.15 \pm 14.48$ & 0.098 \\
\hline ALB & $45.69 \pm 4.11$ & $44.13 \pm 4.10$ & 0.036 \\
\hline URIC & $329.15 \pm 79.71$ & $370.78 \pm 109.79$ & 0.019 \\
\hline $\mathrm{HbA1C}$ & $6.27 \pm 1.21$ & $6.12 \pm 1.08$ & 0.469 \\
\hline Fasting Glucose & 6.332 .13 & $5.79 \pm 2.03$ & 0.152 \\
\hline TG & $1.81 \pm 1.12$ & $1.93 \pm 1.59$ & 0.653 \\
\hline $\mathrm{TC}$ & $4.46 \pm 1.08$ & $4.50 \pm 1.16$ & 0.820 \\
\hline HDL-C & $1.18 \pm 0.34$ & $1.20 \pm 0.38$ & 0.704 \\
\hline LDL-C & $2.74 \pm 0.91$ & $2.74 \pm 0.95$ & 0.970 \\
\hline eGFR & $103.65 \pm 32.28$ & $102.09 \pm 25.30$ & 0.762 \\
\hline
\end{tabular}




\begin{tabular}{|llll|}
\hline & NCSF group $(\mathrm{n}=55)$ & CSF group $(\mathrm{n}=71)$ & $\boldsymbol{p}$ \\
\hline NT-proBNP & $114.53 \pm 143.88$ & $142.57 \pm 196.85$ & 0.419 \\
\hline
\end{tabular}

In the Spearman correlation analyses (Table 3), the $\operatorname{LMR}(r=-0.21, p=0.026), \operatorname{ALB}(r=-0.187, p=0.036)$ level and male sex $(r=-0.265, p=0.003)$ were negatively correlated with CSF, whereas the diameters of the $\operatorname{LAD}(r=0.297, p<0.001), \operatorname{LCX}(r=0.218, p=0.016)$ and $\operatorname{RCA}(r=0.235, p=0.01)$, as well as the mean diameter of the coronary arteries $(r=0.337, p<0.001), \operatorname{NLR}(r=0.245, p=0.009)$ and URIC $(r=0.218, p=$ $0.021)$ level were positively correlated with CSF. Smoking history $(r=0.2, p=0.026)$ was also positively correlated with CSF.

Table 3

Spearman's rho correlation analysis between the CSF with risk factors

\begin{tabular}{|lll|}
\hline Variable & $\mathbf{r}$ & $\boldsymbol{p}$ \\
\hline Gender & -0.265 & 0.003 \\
\hline Smoking history & 0.2 & 0.026 \\
\hline LAD diameter $(\mathrm{mm})$ & 0.297 & $<0.001$ \\
\hline LCX diameter $(\mathrm{mm})$ & 0.218 & 0.016 \\
\hline RCA diameter $(\mathrm{mm})$ & 0.235 & 0.01 \\
\hline Mean D (mm) & 0.337 & $<0.001$ \\
\hline NLR & 0.245 & 0.009 \\
\hline LMR & -0.21 & 0.026 \\
\hline URIC & 0.218 & 0.021 \\
\hline ALB & -0.187 & 0.036 \\
\hline
\end{tabular}

To further explore the independent predictor(s) of CSF, univariable and multivariable logistic regression model analyses were performed based on the correlation analysis results (Table 4). Because the diameters of the LAD and LCX were components of the Mean $D$, we thought that it might negatively affect the regression analysis results. Therefore, we didn't evaluate the diameters of the LAD and LCX in the multivariable regression analysis. Collinearity diagnostics showed that the VIF of males, smoking history, mean diameter of coronary arteries, NLR, LMR, ALB level and URIC level were less than 10 . We performed the multivariate analysis by using the backward LR method. Male sex(odds ratio(OR):1.601,95\%Cl:0.443$5.781, p=0.437)$, smoking history (OR:1.478, 95\% Cl: 0.505-4.324, $p=0.476)$,

NLR(OR:1.741,95\%Cl:0.854-3.549, $p=0.127)$ and ALB(OR:0.917,95\%Cl:0.826-1.019, $p=0.107)$ level were 
not found to be independent predictors for CSF phenomenon. Decreased LMR (OR: $0.614 ; 95 \%$ Cl: $0.464-$ $0.814 ; p=0.001$ ), increased mean diameter of coronary arteries (OR: $2.634 ; 95 \% \mathrm{Cl}: 1.54-4.51 ; p<0.001$ ) and URIC level (OR: $1.006 ; 95 \% \mathrm{Cl}: 1.001-1.012 ; p=0.018)$ were found to be independent predictors of CSF development.

Table 4

Univariable and multivariable logistic regression analyses on the presence of slow coronary flow

\begin{tabular}{|lll|}
\hline Variables & Univariable & Multivariable \\
\cline { 2 - 3 } & OR $(95 \% \mathrm{Cl}) \boldsymbol{p}$ & OR $(95 \% \mathrm{Cl}) \boldsymbol{p}$ \\
\hline Male & $3.369(1.451-7.820) 0.005$ & $1.601(0.443-5.781) 0.437$ \\
\hline Smoking history & $2.236(0.218-0.918) 0.028$ & $1.478(0.505-4.324) 0.476$ \\
\hline LAD diameter & $1.584(1.169-2.147) 0.003$ & \\
\hline LCX diameter & $1.531(1.169-2.005) 0.002$ & \\
\hline Mean diameter of coronary arteries & $2.62(1.543-4.117)<0.001$ & $2.634(1.54-4.51)<0.001$ \\
\hline URIC & $1.005(1.001-1.009) 0.023$ & $1.006(1.001-1.012) 0.018$ \\
\hline NLR & $2.18(1.321-3.599) 0.002$ & $1.741(0.854-3.549) 0.127$ \\
\hline LMR & $0.67(0.530-0.847) 0.001$ & $0.614(0.464-0.814) 0.001$ \\
\hline ALB & $0.911(0.833-0.995) 0.039$ & $0.917(0.826-1.019) 0.107$ \\
\hline OR: odds ratio & & \\
\hline
\end{tabular}

The ROC curve analysis demonstrated that the specificity of the Mean $D>5.76 \mathrm{~mm}$ in predicting CSF was $70.59 \%$ and the sensitivity was $64.81 \%$ (AUC $=0.696, p<0.001$ ). It was revealed that using a cutoff level of LMR $<4.11$ predicted CSF with a sensitivity of $90.9 \%$ and specificity of $40.85 \%(A U C=0.698, p<0.001)$. In addition, the URIC level was found to have the area under the curve (AUC $=0.616, p=0.026)$ with an optimal URIC cutoff value of $314.39 \mu \mathrm{mol} / \mathrm{L}$ (sensitivity $74.65 \%$, specificity $49.09 \%$ ). In addition, the combination revealed a cutoff level of $\leq-4.08$ with a sensitivity of $86.76 \%$ and specificity of $61.11 \%$ (AUC $=0.772, p<0.001)$. Combine Mean D,URIC and LMR to produce a new predictor, the combination(AUC = $0.772, p<0.001$ ) with an optimal cutoff value of less than -4.08 with a sentivity of $86.76 \%$ and speciticity of $61.11 \%$. Comparison of four ROC curves by using the method of DeLong et al. was also performed. The predictive performance of the combination was significantly superior to that of Mean $\mathrm{D}(p$ $=0.0053)$ and URIC level $(p=0.0266)$; however, the difference between the areas of combination and LMR was not significant $(p=0.1061)$. 


\section{Discussion}

The main discoveries of this study can be summarized as follows: 1) the inflammatory indicators were higher in the CSF group than in the NCSF group; 2) LMR, mean diameter of coronary arteries and URIC level were independent predictors in predicting CSF phenomenon in patients with isolated CAE; and 3) the predictive power of LMR was not inferior to that of the combination.

As the detailed pathogenesis of CAE and CSF are still uncertain, hypothesis including chronic inflammation, atherosclerosis, endothelial dysfunction and oxidative stress may have participated in the pathological processes of the two diseases ${ }^{[1-12,14]}$. Many studies focused on inflammation; however, other aspects of research were relatively few.

As is known, monocytes and lymphocytes are vital immune cells. They also play an important role in the atherosclerosis process ${ }^{[3,9,10,15-20]}$. In response to the stimulation of inflammatory cytokines, monocytes are recruited into the intima and subintima by the assistance of adhesion molecules during the initial stage $^{[7,17]}$. After migration, monocytes differentiate into macrophages, devouring oxidized LDL-C and releasing a large number of inflammatory factors, such as interleukin(IL)-1, IL-6, tumor necrosis factor (TNF)-a, and macrophage colony stimulating factor, which attract more monocytes ${ }^{[20]}$. Lymphocytes can enhance the immune response by regulating catecholamine and cortisol levels in the anti-inflammatory milieu. However, as catecholamine and cortisol levels increase, lymphocyte numbers will gradually decline. The hypothesis of this phenomenon includes decreased lymphocyte proliferation, lymphocyte apoptosis and differentiation of lymphocytes in the redistribution of lymphoid organs ${ }^{[16,17,20]}$.

Because the LMR combines two kinds of immune cells, it has long been used as an indicator of the cancer patient condition and treatment ${ }^{[21-23]}$. In recent years, studies have found that the LMR is related to stable angina ${ }^{[16,17]}$, acute coronary syndrome $(\mathrm{ACS})^{[24]}$ and $\mathrm{CSF}^{[7]}$, and it even can be used as an independent predictor of major adverse cardiac events (MACE ${ }^{[18,20,24]}$. Yildirim et al ${ }^{[15]}$. found that the expression of active markers on monocyte-derived dendritic cells in patients with CAE was significantly higher compared to that in patients with CAD. Yayla ${ }^{[7]}$ investigated the LMR in CSF and the normal coronary group found that a low LMR was independently associated with CSF. Kalyoncuoglu et al ${ }^{[9]}$. found that a higher monocyte-to-HDL-C ratio (MHR) and lower LMR values were independent predictors of slow flow/no reflow in patients with non-ST-elevated myocardial infarction (NSTEMI). In our study, the LMR was significantly lower in the CSF group and was found to be an independent risk factor for CSF development in isolated CAE.

URIC can impair nitric oxide generation in endothelial cells through multiple pathways, leading to endothelial dysfunction ${ }^{[4,12,25,26]}$. Studies have shown that it can also induce vascular smooth muscle cell (VSMC) proliferation and differentiation by activating mitogen-activated protein kinases ${ }^{[27]}$. VSMCs migrated from the middle layer to the subintima and participated in the formation and development of atherosclerotic plaques. Our study suggested that URIC was an independent predictor of CSF in isolated CAE, speculating that the progress of atherosclerosis was accelerated in this group. Both the LMR and 
URIC level played an exclusive role in the early formation of atherosclerotic plaques and the growth of the lipid core, suggesting that early rapid atherosclerotic formation and development in a population with CAE might be an important factor for CSF.

The deep impression of CAE in angiography is local or diffuse vascular expansion. It is viewed in the cross-section of a coronary artery as circular, according to the flow equation $Q=\pi r^{2 \wedge} v$, where $Q$ is traffic, $r$ is radius, and $\mathrm{v}$ is flow velocity. It is known that when the traffic is constant, flow velocity is inversely proportional with the square of the radius. This was consistent with our study, showing that the Mean D in the CSF group was larger than that in the NCSF group.

Other studies also found that some inflammation indicators relate to CAE or CSF, such as the NLR and albumin level. Yilmaz ${ }^{[11]}$ suggested the NLR was significantly higher in patients with CAE, CSF and CAD compared to those in the normal group and was an independent predictor of these diseases. ALB is also

a kind of negative acute phase protein ${ }^{[1,28]}$. Cetin ${ }^{[1]}$ found that the ALB level was significantly lower in the CSF group than in the normal group and was an independent predictor of CSF. This study also found that both the NLR and albumin level were significantly different in both groups. However, the predictive power of the NLR and albumin level in CSF didn't reach statistical significance. A possible reason might be the different control groups in these studies. The control group in our study was patients with isolated CAE who had a certain level of inflammation, resulting in the prediction ability of inflammation indicators not reaching statistical significance.

The combination of the LMR, URIC and Mean D generated a new combination predictor. Comparison of the areas under the $4 \mathrm{ROC}$ curves suggested the predictive power of the combination was superior to URIC and Mean $D(P<0.001)$, but there was no statistically significant difference with the LMR.

\section{Limitation}

There are some limitations in this study. The first limitation relates to its retrospective design. Some risk factors, such as the matrix metalloproteinase family, tissue inhibitors of metalloproteinase ${ }^{[29,30]}$ and adropin ${ }^{[3]}$, which have a significant role in CAE development, couldn't be collected. Second, multivariable logistic regression analyses were performed to identify independent predictors of CSF in CAE; however, it was impossible to control for unknown confounders. In addition, this study has reviewed the cases in the center over a 10-year period, and due to the low morbidity of both diseases, the sample size was still not large enough to reflect more latent risk factors.

\section{Conclusion}

Patients with CAE combined with CSF have a more significant development of atherosclerosis and serious inflammatory reactions. The LMR, URIC level and Mean D were independent predictors of CSF in $\mathrm{CAE}$, and the predictive power of the LMR was not inferior to the combination. 


\section{List Of Abbrievation}

LMR lymphocyte-to-monocyte ratio

CSF coronary slow flow

CAE coronary artery ectasia

Mean D mean diameter of coronary arteries

URIC uric acid

\section{Declarations}

Ethics approval and consent to participate The study is approved and consent by Fuwai Hospital Ethics Committee.

Consent for publication Not applicable

Availability of data and materials The datasets used and analysed during the current study are available from the corresponding author on reasonable request

Competing interests The authors declare that they have no competing interests

Funding: Clinical and Translational Foundation of The Chinese Academy of Medical Sciences (2019XK320064)

Authors' contributions: ZXY and SBQ contributed to drafting and revising the manuscript. JSY contributed to the clinical data collection and coronary angiography operation. JGC contributed to analysis and interpretation of data. All the authors critically revised the manuscript and gave final approval and agreed to be accountable for all aspects of the work, ensuring both its integrity and accuracy.

Acknowledgements Not applicable

\section{References}

1. Cetin M, Zencir C, Tasolar H, Baysal E, Balli M, Akturk E (2014) The association of serum albumin with coronary slow flow. Wien Klin Wochenschr 126 (15-16):468-473. doi:10.1007/s00508-014-05598.

2. Isik T, Ayhan E, Uyarel H, Tanboga IH, Kurt M, Uluganyan M, Ergelen M, Eksik A (2013) Association of neutrophil to lymphocyte ratio with presence of isolated coronary artery ectasia. Turk Kardiyol Dern Ars 41 (2):123-130. doi:10.5543/tkda.2013.17003

3. Ozkan B, Orscelik O, Yildirim Yaroglu H, Balci S, Ozcan MK, Celik A, Ozcan IT (2019) Association between serum adropin levels and isolated coronary artery ectasia in patients with stable angina 
pectoris. Anatol J Cardiol 22 (5):250-255. doi:10.14744/AnatolJCardiol.2019.90349

4. Sen N, Ozcan F, Uygur B, Aksu T, Akpinar I, Cay S, Cetin M, Sokmen E, Akcakoyun M, Maden O, Balbay Y, Erbay AR (2009) Elevated serum uric acid levels in patients with isolated coronary artery ectasia. Turk Kardiyol Dern Ars 37 (7):467-472

5. Malviya A, Jha PK, Mishra A (2017) Isolated coronary artery ectasia: Clinical, angiographic, and follow up characteristics. Indian Heart J 69 (5):619-623. doi:10.1016/j.ihj.2016.12.017

6. Hawkins BM, Stavrakis S, Rousan TA, Abu-Fadel M, Schechter E (2012) Coronary slow flowprevalence and clinical correlations. Circ J 76 (4):936-942. doi:10.1253/circj.cj-11-0959

7. Yayla C, Akboga MK, Gayretli Yayla K, Ertem AG, Efe TH, Sen F, Unal S, Acar B, Ozcan F, Turak O, Ozeke $O$ (2016) A novel marker of inflammation in patients with slow coronary flow: lymphocyte-tomonocyte ratio. Biomark Med 10 (5):485-493. doi:10.2217/bmm-2016-0022

8. Demirci E, Celik O, Kalcik M, Bekar L, Yetim M, Dogan T (2019) Evaluation of homocystein and asymmetric dimethyl arginine levels in patients with coronary slow flow phenomenon. Interv Med Appl Sci 11 (2):89-94. doi:10.1556/1646.11.2019.07.

9. Kalyoncuoglu M, Biter HI, Ozturk S, Belen E, Can MM (2020) Predictive accuracy of lymphocyte-tomonocyte ratio and monocyte-to-high-density-lipoprotein-cholesterol ratio in determining the slow flow/no-reflow phenomenon in patients with non-ST-elevated myocardial infarction. Coron Artery Dis 31 (6):518-526. doi:10.1097/MCA.0000000000000848.

10. Kurtul A, Yarlioglues M, Celik IE, Duran M, Elcik D, Kilic A, Oksuz F, Murat SN (2015) Association of lymphocyte-to-monocyte ratio with the no-reflow phenomenon in patients who underwent a primary percutaneous coronary intervention for ST-elevation myocardial infarction. Coron Artery Dis 26 (8):706-712. doi:10.1097/MCA.0000000000000301

11. Yilmaz M, Korkmaz H, Bilen MN, Uku O, Kurtoglu E (2016) Could neutrophil/lymphocyte ratio be an indicator of coronary artery disease, coronary artery ectasia and coronary slow flow? J Int Med Res 44 (6):1443-1453. doi:10.1177/0300060516664637.

12. Demir S, Karakoyun G, Kanadasi M (2014) Elevated high sensitivity C-reactive protein and uric acid levels in coronary artery ectasia. Acta Biochim Pol 61 (4):687-691

13. Gibson CM, Cannon CP, Daley WL, Dodge JT Jr, Alexander B Jr, Marble SJ, et al. TIMI frame count: A quantitative method of assessing coronary artery flow. Circulation 1996; 93: 879-888.

14. Brunetti ND, Salvemini G, Cuculo A, Ruggiero A, De Gennaro L, Gaglione A, Di Biase M (2014) Coronary artery ectasia is related to coronary slow flow and inflammatory activation. Atherosclerosis 233 (2):636-640. doi:10.1016/j.atherosclerosis.2014.01.018.

15. Yildirim N, Tekin IO, Dogan SM, Aydin M, Gursurer M, Cam F, Gungorduk A, Akoz A (2007) Expression of monocyte and lymphocyte adhesion molecules is increased in isolated coronary artery ectasia. Coron Artery Dis 18 (1):49-53. doi:10.1097/MCA.0b013e32801104d4.

16. Gong S, Gao X, Xu F, Shang Z, Li S, Chen W, Yang J, Li J (2018) Association of lymphocyte to monocyte ratio with severity of coronary artery disease. Medicine (Baltimore) 97 (43):e12813. doi:10.1097/MD.0000000000012813 
17. Kose N, Akin F, Yildirim T, Ergun G, Altun I (2019) The association between the lymphocyte-tomonocyte ratio and coronary artery disease severity in patients with stable coronary artery disease. Eur Rev Med Pharmacol Sci 23 (6):2570-2575. doi:10.26355/eurrev_201903_17406.

18. Kiris T, Celik A, Varis E, Akan E, Akyildiz ZI, Karaca M, Nazli C, Dogan A (2017) Association of Lymphocyte-to-Monocyte Ratio With the Mortality in Patients With ST-Elevation Myocardial Infarction Who Underwent Primary Percutaneous Coronary Intervention. Angiology 68 (8):707-715. doi:10.1177/0003319716685480.

19. Si Y, Liu J, Shan W, Zhang Y, Han C, Wang R, Sun L (2020) Association of lymphocyte-to-monocyte ratio with total coronary plaque burden in patients with coronary artery disease. Coron Artery Dis 31 (7):650-655. doi:10.1097/MCA.0000000000000857.

20. Wang Q, Ma J, Jiang Z, Wu F, Ping J, Ming L (2017) Association of lymphocyte-to-monocyte ratio with in-hospital and long-term major adverse cardiac and cerebrovascular events in patients with STelevated myocardial infarction. Medicine (Baltimore) 96 (34):e7897. doi:10.1097/MD.0000000000007897.

21. Tan D, Fu Y, Tong W, Li F. Prognostic significance of lymphocyte to monocyte ratio in colorectal cancer: A meta-analysis. Int J Surg. 2018 Jul;55:128-138. doi: 10.1016/j.ijsu.2018.05.030. Epub 2018 May 26. PMID: 29807167.

22. Tan D, Fu Y, Tong W, Li F. Prognostic significance of lymphocyte to monocyte ratio in colorectal cancer: A meta-analysis. Int J Surg. 2018 Jul;55:128-138. doi: 10.1016/j.ijsu.2018.05.030. Epub 2018 May 26. PMID: 29807167.

23. Pan YC, Jia ZF, Cao DH, Wu YH, Jiang J, Wen SM, Zhao D, Zhang SL, Cao XY. Preoperative lymphocyte-to-monocyte ratio (LMR) could independently predict overall survival of resectable gastric cancer patients. Medicine (Baltimore). 2018 Dec;97(52):e13896. doi: 10.1097/MD.0000000000013896. PMID: 30593200; PMCID: PMC6314713.

24. Quan XQ, Wang RC, Zhang Q, Zhang CT, Sun L (2020) The predictive value of lymphocyte-tomonocyte ratio in the prognosis of acute coronary syndrome patients: a systematic review and metaanalysis. BMC Cardiovasc Disord 20 (1):338. doi:10.1186/s12872-020-01614-x.

25. Kang DH, Han L, Ouyang X, Kahn AM, Kanellis J, Li P, Feng L, Nakagawa T, Watanabe S, Hosoyamada M, Endou H, Lipkowitz M, Abramson R, Mu W, Johnson RJ (2005) Uric acid causes vascular smooth muscle cell proliferation by entering cells via a functional urate transporter. Am J Nephrol 25 (5):425-433. doi:10.1159/000087713.

26. Kanbay M, Segal M, Afsar B, Kang DH, Rodriguez-Iturbe B, Johnson RJ (2013) The role of uric acid in the pathogenesis of human cardiovascular disease. Heart 99 (11):759-766. doi:10.1136/heartjnl2012-302535.

27. Kanellis J, Watanabe S, Li JH, Kang DH, Li P, Nakagawa T, Wamsley A, Sheikh-Hamad D, Lan HY, Feng L, Johnson RJ (2003) Uric acid stimulates monocyte chemoattractant protein-1 production in vascular smooth muscle cells via mitogen-activated protein kinase and cyclooxygenase-2. Hypertension 41 (6):1287-1293. doi:10.1161/01.HYP.0000072820.07472.3B. 
28. Arques S (2018) Human serum albumin in cardiovascular diseases. Eur J Intern Med 52:8-12. doi:10.1016/j.ejim.2018.04.014.

29. Liu R, Chen L, Wu W, Chen H, Zhang S. Extracellular matrix turnover in coronary artery ectasia patients. Heart Vessels. 2016 Mar;31(3):351-9. doi: 10.1007/s00380-014-0622-4. Epub 2015 Jan 10. PMID: 25576491.

30. Dahi S, Karliner JS, Sarkar R, Lovett DH. Transgenic expression of matrix metalloproteinase-2 induces coronary artery ectasia. Int J Exp Pathol. 2011 Feb;92(1):50-6. doi: 10.1111/j.13652613.2010.00744.x. Epub 2010 Oct 29. PMID: 21039989; PMCID: PMC3052756.

\section{Figures}

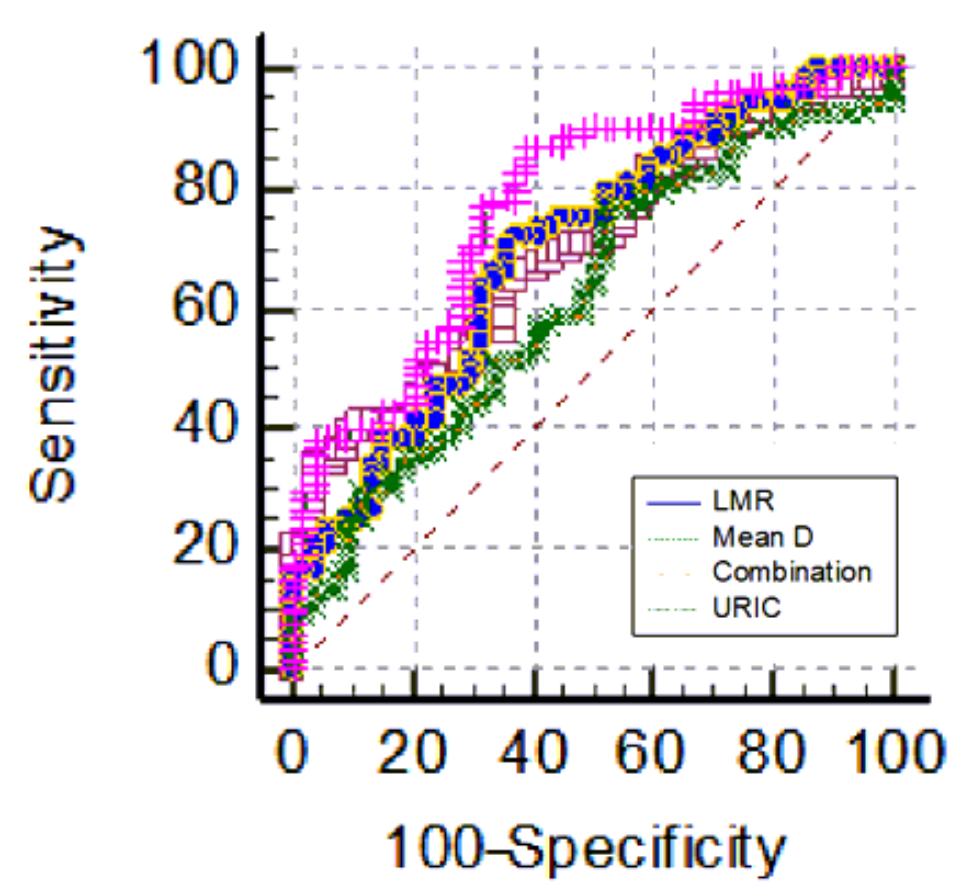

\begin{tabular}{lccccc}
\hline & Difference between areas & $95 \% \mathrm{Cl}$ & Z-statistic & & $P$ \\
\cline { 2 - 3 } \cline { 5 - 6 } LMR vs Mean D & 0.00231 & -0.129 to 0.134 & 0.0346 & 0.97 \\
LMR vs URIC & 0.0728 & -0.0748 to 0.220 & 0.967 & 0.33 \\
URIC vs Mean D & 0.0752 & -0.0614 to 0.212 & 1.079 & 0.28 \\
Combination vs Mean D & 0.0757 & 0.0225 to 0.129 & 2.789 & 0.0053 \\
Combination vs URIC & 0.151 & 0.0175 to 0.284 & 2.217 & 0.0266 \\
Combination vs LMR & 0.078 & -0.0166 to 0.173 & 1.616 & 0.1061 \\
\hline
\end{tabular}

\section{Figure 1}

Comparison of the predictive powers of the mean diameter of coronary arteries (Mean D), uric acid (URIC), lymphocyte-to-monocyte ratio (LMR) and their combination in predicting the development of 


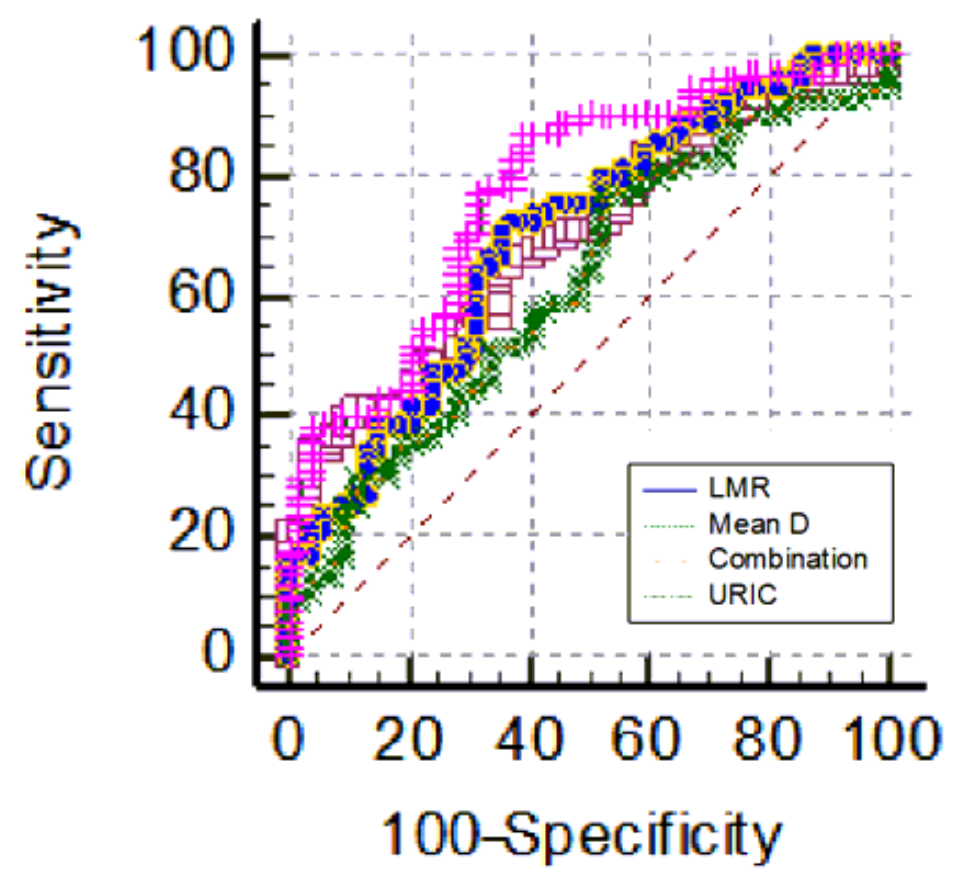

\begin{tabular}{lccccc}
\hline & Difference between areas & & $95 \% \mathrm{Cl}$ & Z-statistic & $P$ \\
\cline { 2 - 3 } \cline { 5 - 6 } LMR vS Mean D & 0.00231 & -0.129 to 0.134 & 0.0346 & 0.97 & 0.33 \\
LMR vs URIC & 0.0728 & -0.0748 to 0.220 & 0.967 & 0.28 \\
URIC vs Mean D & 0.0752 & -0.0614 to 0.212 & 1.079 & 0.0053 \\
Combination vs Mean D & 0.0757 & 0.0225 to 0.129 & 2.789 & 0.0266 \\
Combination vs URIC & 0.151 & 0.0175 to 0.284 & 2.217 & 0.1061 \\
Combination vs LMR & 0.078 & -0.0166 to 0.173 & 1.616 & 0.16 \\
\hline
\end{tabular}

\section{Figure 1}

Comparison of the predictive powers of the mean diameter of coronary arteries (Mean D), uric acid (URIC), lymphocyte-to-monocyte ratio (LMR) and their combination in predicting the development of coronary slow flow phenomenon. 